Kragujevac Journal of Mathematics

Volume 42(3) (2018), Pages 325-333.

\title{
TREES WITH THE MINIMAL SECOND ZAGREB INDEX
}

\author{
M. ELIASI ${ }^{1}$ AND A. GHALAVAND ${ }^{1}$
}

\begin{abstract}
For simple graph $G$ with edge set $E(G)$, the second Zagreb index of $G$ is defined as $M_{2}(G)=\sum_{u v \in E(G)}\left[d_{G}(u) d_{G}(v)\right]$, where $d_{G}(v)$ is the degree of the vertex $v$ in $G$. In this paper, we identify the nine classes of trees, which have the first to the sixth smallest second Zagreb indices, among all the trees of the order $n \geq 11$.
\end{abstract}

\section{INTRODUCTION}

Let $G$ be a finite, connected, simple graph (an undirected graph containing no loops and no multiple edges) with the vertex set $V(G)$ and edge set $E(G)$. For vertex $v \in V(G)$, we denote the degree of $v$ by $d_{G}(v)$ and the set of vertices adjacent to $v$ by $N_{G}(v)$, respectively. The maximum vertex degree is denoted by $\Delta=\Delta(G)$. A pendent vertex of $G$ is a vertex with degree one. If $G$ is acyclic graph, then $G$ is called a tree. Any tree with at least two vertices has at least two pendent vertices. The second Zagreb index, $M_{2}(G)$, is defined as follows:

$$
M_{2}(G)=\sum_{u v \in E(G)}\left[d_{G}(u) d_{G}(v)\right] .
$$

The second Zagreb index is a topological index; real numbers related to a graph, which are invariant under graph isomorphisms. Topological indices have many applications in chemistry, biology, and other branches of science [5]. The second Zagreb index was found to occur in certain approximate expressions of the total $\pi$-electron energy of alternant hydrocarbons [7]. We encourage the reader to consult $[2,13,19,20]$ and [21] for the historical background, computational techniques, and mathematical properties of the second Zagreb index. Recently, a search for graphs in different classes with extreme values of the second Zagreb index has been conducted by many researchers,

Key words and phrases. Second Zagreb index, subdivision, tree.

2010 Mathematics Subject Classification. Primary: 05C07. Secondary: 05C05.

Received: September 20, 2016.

Accepted: March 15, 2017. 
see $[12,17]$. Goubko [6] described trees with the minimal second Zagreb index as among those with a fixed number of pendent vertices. Deng [4] presented a unified and simplified approach to the largest and smallest second Zagreb indices for trees, unicyclic graphs, and bicyclic graphs by introducing some transformations. In [1], the authors characterized trees with maximal and minimal $M_{2}$ as among those with a given number of segments or a given number of branching vertices. Vukicevic et al. [16] reported a simple approach to calculate the maximal value of $M_{2}$ for trees with a fixed number of vertices of a given degree. The extreme $M_{2}$ values of graphs with connectivity at most $k$, were obtained in [11,18], and the $M_{2}$ bounds of unicyclic graphs were obtained by Yan et al. [17]. The sharp $M_{2}$ bounds of bipartite graphs with a given diameter were obtained in $[9,10]$. Lang et al. [9] gave a necessary condition for a bipartite graph to attain the maximal value of the second Zagreb index. Further, Das and Gutman proved that the connected $n$-vertex graph with minimum second Zagreb index is an $n$-vertex tree and they demonstrated that the tree with minimum value of $M_{2}$ represents the path [3]. In [8], the first and second maximum values of the second Zagreb index in class of tetracyclic $n$-vertex graphs was presented.

In the above references, the authors obtained the first and second maximal $M_{2}$ or the first minimal $M_{2}$ in some classes of graphs. In this paper, we identify the nine classes of trees, which have the first to the sixth smallest second Zagreb indices among all the trees of the order $n \geq 11$.

\section{Preliminaries}

For subset $E$ of $E(G)$, we denote the subgraph of $G$ obtained by deleting the edges of $E$ by $G-E$. If $E=\{u v\}$, the subgraphs $G-E$ will be written as $G-u v$ for short. In addition, for any two nonadjacent vertices $x$ and $y$ of graph $\mathrm{G}$, let $G+x y$ be the graph obtained from $G$ by adding an $x y$ edge. The set of all $n$-vertex trees will be denoted by $\tau(n)$. We denote the path graph and the star graph (both with $n$ vertices) by $P_{n}$ and $S_{n}$, respectively. A non-increasing sequence $\left(d_{1}, d_{2}, \ldots, d_{n}\right)$ of nonnegative integers is said to be graphic if a finite simple graph $G$ exists, with vertices $v_{1}, v_{2}, \ldots, v_{n}$, such that each $v_{i}$ has degree $d_{i}$.

Further, suppose that $\left(d_{1}, d_{2}, \ldots, d_{n}\right)=(\underbrace{x_{1}, \ldots, x_{1}}_{\alpha_{1} \text { times }}, \underbrace{x_{2}, \ldots, x_{2}}_{\alpha_{2} \text { times }}, \ldots, \underbrace{x_{t}, \ldots, x_{t}}_{\alpha_{t} \text { times }})$, where $d_{1}=x_{1}>x_{2}>\ldots>x_{t}=d_{n}$ and $\alpha_{1}, \ldots, \alpha_{t}$ are positive integers, such that $\alpha_{1}+\alpha_{2}+\ldots+\alpha_{t}=n$. Then, we write, $\left(d_{1}, d_{2}, \ldots, d_{n}\right)=\left(x_{1}^{\alpha_{1}}, x_{2}^{\alpha_{2}}, \ldots, x_{t}^{\alpha_{t}}\right)$. In addition, $T\left(x_{1}^{\alpha_{1}}, \ldots, x_{m}^{\alpha_{m}}\right)$ represents the class of trees with $\alpha_{i}$ vertices of the degree $x_{i}, i=1, \ldots, m$.

A rooted tree is a tree with a designated vertex called a root. If vertex $v$ immediately precedes vertex $w$ on the path from the root to $w$, then $v$ is a parent of $w$ and $w$ is child of $v$. A leaf in a rooted tree is any vertex with no children and a vertex of degree 1. Vertices with the same parent are called siblings. Vertex $w$ is called a descendant of vertex $v$ if $v$ is on the unique path from the root to $w$. 
The subdivision of graph $G$ is a new graph obtained by adding vertices inside the edges.

For a positive number $n \geq 11$, let:

$$
\begin{aligned}
T_{9}(n)= & \left\{T \in T\left(4^{1}, 2^{n-5}, 1^{4}\right) \mid m_{1,4}(T)=3, m_{1,2}(T)=m_{2,4}(T)=1 \text { and } m_{2,2}(T)\right. \\
& =n-6\} \\
T_{12}(n)= & \left\{T \in T\left(3^{3}, 2^{n-8}, 1^{5}\right) \mid m_{1,3}(T)=5, m_{1,2}(T)=m_{3,3}(T)=0, m_{2,3}(T)=4\right. \\
& \text { and } \left.m_{2,2}(T)=n-10\right\},
\end{aligned}
$$

where $m_{i, j}(T)$ is the number of edges of $T$ connecting the vertices of degrees $i$ and $j$. In Figure 1, we illustrate the trees in $T_{12}(n)$, the $B B$ tree.

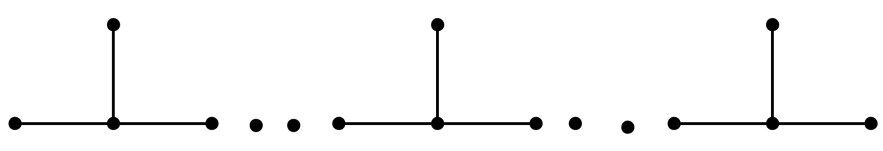

$B B$

Figure 1. $B B$ tree

For a graph $G$ with $n \geq 3$ vertices and $m$ edges, denote the number of vertices with degree $i$ for $i=1,2, \ldots, n-1$, by $n_{i}$. For $1 \leq i \leq j \leq n-1$, when no confusion can arise, we shall simply write $m_{i, j}$ instead of $m_{i, j}(G)$. Now we have:

$$
\begin{aligned}
n & =n_{1}+n_{2}+\cdots+n_{n-1} \\
2 m & =n_{1}+2 n_{2}+\cdots+(n-1) n_{n-1} \\
n_{1} & =m_{1,2}+m_{1,3}+\cdots+m_{1, n-1} \\
2 n_{2} & =m_{1,2}+2 m_{2,2}+\cdots+m_{2, n-1}
\end{aligned}
$$

$$
\begin{aligned}
(n-1) n_{n-1} & =m_{1, n-1}+m_{2, n-1}+\cdots+2 m_{n-1, n-1} . \\
M_{2}(G) & =\sum_{1 \leq i \leq j \leq n-1} m_{i, j} . i . j
\end{aligned}
$$

where the last equality was given by Vukicevic et al. [16]. Note that if $G \in \tau(n)$, then $m=n-1$ and $n_{1} \geq 2$. We start with the following lemma.

Lemma 2.1. Let $u$ be the root of rooted tree $T$ and $d_{T}(u) \geq 3$. If $v_{0} v_{1}$ is a pendent edge in $T\left(d_{T}\left(v_{0}\right) \geq 2, d_{T}\left(v_{1}\right)=1\right)$, then for each $u_{1} \in V(T)$, in which $u_{1}$ and $v_{0}$ do not have same parent and $d_{T}(u) \geq d_{T}\left(v_{0}\right), M_{2}\left(T-u u_{1}+v_{1} u_{1}\right)<M_{2}(T)$ (Figure 2). 


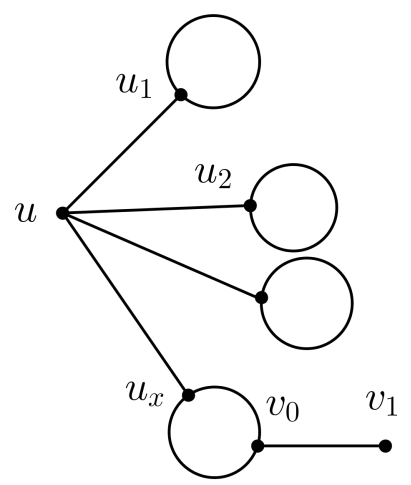

$T$

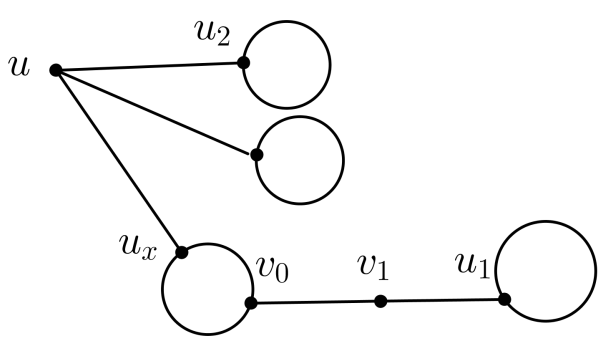

$T_{1}$

Figure 2. Trees $T$ and $T_{1}$ in Lemma 2.1

Proof. Suppose that $T_{1}=T-u u_{1}+v_{1} u_{1}, d_{T}(u)=x, N_{T}(u)=\left\{u_{1}, \ldots, u_{x}\right\}$ and $d_{T}\left(u_{i}\right)=d_{i}$, for $i=1, \ldots, x$ (Figure 2). If $u \neq v_{0}$, then

$$
\begin{aligned}
M_{2}(T)-M_{2}\left(T_{1}\right)= & 1 d_{T}\left(v_{0}\right)+d_{T}(u) d_{T}\left(u_{1}\right)+\sum_{i=2}^{x} d_{T}(u) d_{i} \\
& -2 d_{T}\left(v_{0}\right)-2 d_{T}\left(u_{1}\right)-\sum_{i=2}^{x}\left[d_{T}(u)-1\right] d_{i} \\
= & -d_{T}\left(v_{0}\right)+\left(d_{T}(u)-2\right) d_{T}\left(u_{1}\right)+\sum_{i=2}^{x} d_{i} \\
\geq & -d_{T}\left(v_{0}\right)+\left(d_{T}(u)-2\right) d_{T}\left(u_{1}\right)+d_{T}(u) \\
\geq & \left(d_{T}(u)-2\right) d_{T}\left(u_{1}\right)>0,
\end{aligned}
$$

because $d_{x} \geq 2$ and $d_{i} \geq 1$ for $2 \leq i \leq x-1$.

Now, suppose that $u=v_{0}$. Without the loss of generality, we may assume that $u_{x}=v_{1}$. Since $d_{T}(u) \geq 3$, then:

$$
\begin{aligned}
M_{2}(T)-M_{2}\left(T_{1}\right)= & d_{T}(u) d_{T}\left(u_{1}\right)+1 d_{T}(u)+\sum_{i=2}^{x-1} d_{T}(u) d_{i} \\
& -\sum_{i=2}^{x-1}\left[d_{T}(u)-1\right] d_{i}-2\left(d_{T}(u)-1\right)-2 d_{T}\left(u_{1}\right) \\
= & -d_{T}(u)+2+d_{T}(u) d_{T}\left(u_{1}\right)-2 d_{T}\left(u_{1}\right)+\sum_{i=2}^{x-1} d_{i} \\
\geq & -d_{T}(u)+2+d_{T}(u) d_{T}\left(u_{1}\right)-2 d_{T}\left(u_{1}\right)+\left(d_{T}(u)-2\right) \\
= & \left(d_{T}(u)-2\right) d_{T}\left(u_{1}\right)>0 .
\end{aligned}
$$

This completes the proof. 


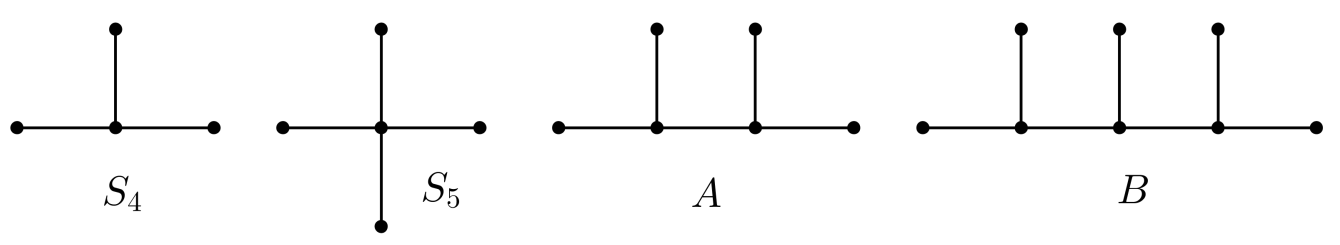

Figure 3 . Trees $S_{4}, S_{5}, A$ and $B$

Remark 2.1. Considering the proof of Lemma 2.1, one can see that if $d_{T}(u)=3$ and $d_{T}\left(v_{0}\right)=4$, then $M_{2}\left(T_{1}\right) \leq M_{2}(T)$.

In Figure 3 we illustrate the trees $S_{4}, S_{5}, A$ and $B$ that we need in the remainder of this paper.

Lemma 2.2. Among all the subdivisions of $S_{5}$, with $n \geq 10$ vertices, the tree obtained by adding all the new vertices to one edge has the minimal second Zagreb index. If $T \in T\left(4^{1}, 2^{n-5}, 1^{4}\right)$, then $M_{2}(T) \geq 4 n-2$. Equality holds if and only if $T \in T_{9}(n)$.

Proof. Let $T$ be a subdivision graph of $S_{5}$, with $n \geq 11$ vertices. Then, $m=n-1, n_{1}=$ 4 and $n_{4}=1$. In addition, since $n \geq 11, m_{1,2}, m_{2,2}$ and $m_{2,4}$ are nonzero integers. By considering equation (2.1), we must obtain the minimum $2 m_{1,2}+4 m_{2,2}+4 m_{1,4}+8 m_{2,4}$ with respect to

$$
\begin{aligned}
n & =n_{2}+5, \\
4 & =m_{1,2}+m_{1,4}, \\
2 n_{2} & =m_{1,2}+2 m_{2,2}+m_{2,4}, \\
4 & =m_{1,4}+m_{2,4} .
\end{aligned}
$$

Therefore, $m_{1,4}=4-m_{1,2}, m_{2,4}=m_{1,2}$, and $m_{2,2}=n-5-m_{1,2}$. So, $M_{2}(T)=$ $4 n-4+2 m_{12}$. Hence, if $m_{1,2}=1$, then the minimum value of $M_{2}$ is obtained. In this case, $m_{1,4}=3, m_{2,4}=1$, and thus $m_{2,2}(T)=n-6$.

Lemma 2.3. Among all the subdivisions of $B$, with $n \geq 11$ vertices, the trees in $T_{12}(n)$ have the minimal second Zagreb index. Moreover, if $T \in T\left(3^{3}, 2^{n-8}, 1^{5}\right)(a$ subdivision graph of $B)$, then $M_{2}(T) \geq 4 n-1$. Equality holds if and only if $T$ is a $B B$ tree.

Proof. Let $T$ be a subdivision graph of $B$, with $n \geq 11$ vertices. Then, $m=n-1$, $n_{1}=5$, and $n_{3}=3$. By considering equation (2.1), we must obtain the minimum $2 m_{1,2}+3 m_{1,3}+4 m_{2,2}+6 m_{2,3}+9 m_{3,3}$ with respect to

$$
\begin{aligned}
n & =n_{2}+8, \\
5 & =m_{1,2}+m_{1,3}, \\
2 n_{2} & =m_{1,2}+2 m_{2,2}+m_{2,3}, \\
9 & =m_{1,3}+m_{2,3}+2 m_{3,3} .
\end{aligned}
$$


Therefore, $m_{1,3}=5-m_{1,2}, m_{2,2}=n-10-m_{1,2}+m_{3,3}$, and $m_{2,3}=4+m_{1,2}-2 m_{3,3}$. So, $M_{2}(T)=4 n-1+m_{1,2}+m_{3,3}$. Hence, if $m_{1,2}=0=m_{3,3}$, then the minimum value of $M_{2}$ is obtained. In this case, $m_{1,3}=5, m_{2,3}=4$, and thus $m_{2,2}=n-10$.

\section{Main Results}

Theorem 3.1. Let $\dot{T}$ be a tree in $\tau(n)$, where $n \geq 11$. If $\Delta(\dot{T}) \geq 4$ and $\dot{T} \notin T_{9}(n)$, then for each $T \in T_{9}(n)$, we have $M_{2}(T)<M_{2}(T)$.

Proof. By induction on $\Delta(\dot{T})$. Suppose that $\Delta\left(T^{\prime}\right)=4$. Let $U_{4}=\left\{u \in V\left(T^{\prime}\right) \mid\right.$ $\left.d_{\hat{T}}(u)=4\right\}$ and fix $u_{0} \in U_{4}$. If $U_{4} \backslash\left\{u_{0}\right\} \neq \emptyset$, then by the repeated application of Lemma 2.1 on the vertices in $U_{4} \backslash\left\{u_{0}\right\}$, $\left(\left|U_{4}\right|-1\right)$-times, we arrive at tree $T_{m}$, in which $u_{0}$ is the only vertex of degree 4 in $V\left(T_{m}\right)$ and in which $M_{2}\left(T_{m}\right)<M_{2}(\dot{T})$. In addition, if $u \in V\left(T_{m}\right)$ and $u \neq u_{0}$, then $d_{T_{m}}(u) \leq 3$. Therefore, without the loss of generality, we suppose that $u_{0}$ is the only vertex of degree 4 in $T$ and that the other vertices have degree almost 3 . We consider the following cases.

Case 1: $n_{3}(\dot{T})=0$. Then, $\dot{T}$ is a subdivision graph of $S_{5}$, and by Lemma 2.2, $M_{2}(T)<M_{2}(\dot{T})$.

Case 2: $n_{3}\left(\dot{T}^{\prime}\right)>0$. Let $U_{3}=\left\{u \in V\left(\hat{T}^{\prime}\right) \mid d_{\hat{T}}(u)=3\right\}$. Suppose that $u \in U_{3}$ and $N_{\hat{T}}(u)=\left\{u_{1}, u_{2}, u_{3}\right\}$, such that $u$ is the root of $T$ and $u_{2}$ is $u_{0}$ or $u_{2}$ is an ancestor of $u_{0}$. Then, according to Remark 2.1, if $v_{1}$ is a leaf in $\dot{T}$ such that $u_{3}$ is an ancestor of $v_{1}$, then $M_{2}\left(\dot{T}-u u_{1}+v_{1} u_{1}\right) \leq M_{2}(\dot{T})$. By the repeated application of this process on the vertices in $U_{3}$, we arrive at a tree $T_{m}$, in which $M_{2}\left(T_{m}\right) \leq M_{2}\left(T^{\prime}\right), n_{3}\left(T_{m}\right)=0$ and $u_{0}$ is the only vertex of degree 4 in $T_{m}$. Therefore, by Case $1, M_{2}(T)<M_{2}\left(T_{m}\right) \leq M_{2}(\dot{T})$.

Now, suppose that $\Delta\left(\dot{T}^{\prime}\right)>4$ and $U_{\Delta(\dot{T})}=\left\{u \in V(\dot{T}) \mid d_{\dot{T}}(u)=\Delta\left(\dot{T}^{\prime}\right)\right\}$. By the repeated application of Lemma 2.1 on the vertices in $U_{\Delta\left(T^{\prime}\right)},\left|U_{\Delta\left(T^{\prime}\right)}\right|$-times, we arrive at a tree $T_{s}$, with $\Delta\left(T_{s}\right)=\Delta(\dot{T})-1$ and $M_{2}\left(T_{s}\right)<M_{2}(\dot{T})$. By the induction hypothesis, $M_{2}(T)<M_{2}\left(T_{s}\right)$, and hence $M_{2}(T)<M_{2}(\dot{T})$.

Theorem 3.2. Let $\dot{T}$ be a tree with $\Delta(\hat{T})=3$, such that the number of its vertices of degree 3 is at least 3 . Then, for each $T \in T_{12}(n)$, we have $M_{2}(T) \leq M_{2}(\dot{T})$.

Proof. We consider the following cases.

Case 1: the number of vertices of degree 3 in $T^{\prime}$ is equal to 3 . Since $\Delta(\dot{T})=3, T^{\prime}$ is a subdivision graph of $B$. Thus, by Lemma $2.3, M_{2}(T) \leq M_{2}(\dot{T})$.

Case 2: $n_{3}\left(T^{\prime}\right) \geq 4$. Let $u_{1}, u_{2}$ and $u_{3}$ be distinct vertices of degree 3 in $T^{\prime}$ and let $U_{3}$ be the set of all vertices of degree 3 in $T$. If $u \in U_{3} \backslash\left\{u_{1}, u_{2}, u_{3}\right\}$, then by Lemma 2.1, we obtain tree $T_{1}$, in which $d_{T_{1}}(u)=2$, and $M_{2}\left(T_{1}\right) \leq M_{2}(T)$. By repeating this argument $\left(\left|U_{3}\right|-4\right)$-times, we obtain tree $T_{s}$, in which $\Delta\left(T_{s}\right)=3, n_{3}\left(T_{s}\right)=3$, and $M_{2}\left(T_{s}\right) \leq M_{2}(\dot{T})$. Case 1 shows that $M_{2}(T) \leq M_{2}\left(T_{s}\right)$. Therefore, $M_{2}(T) \leq$ $M_{2}\left(T^{\prime}\right)$.

Let $T \in \tau(n), T_{9} \in T_{9}(n)$, and $n \geq 11$. We consider the following cases. 


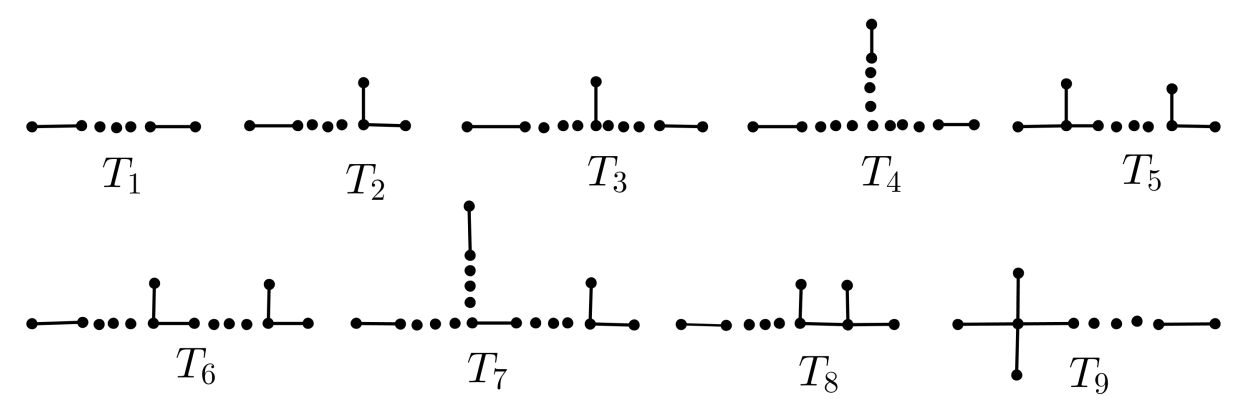

FiguRE 4. Trees in Theorem 3.3

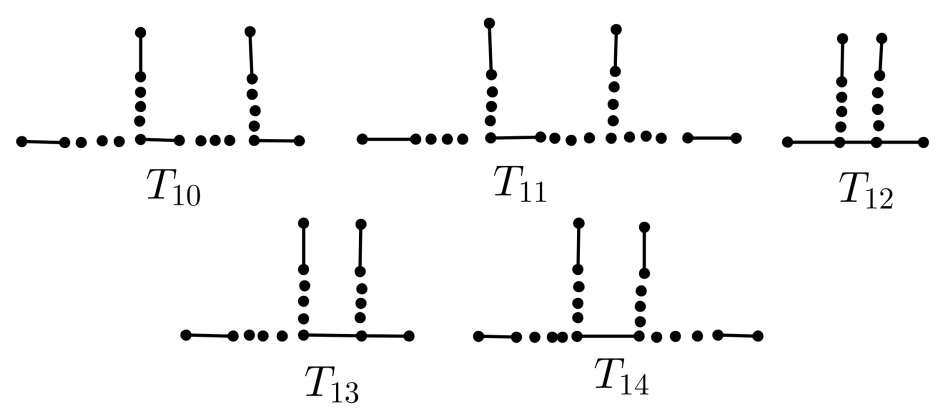

FIGURE 5. Some subdivision graphs of $A$

Case 1: $\Delta(T) \geq 4$. Then, by Theorem 3.1, $M_{2}\left(T_{9}\right) \leq M_{2}(T)$.

Case 2: $\Delta(T)=3$. If $n_{3}(T) \geq 3$, then Theorem 3.2, shows that $M_{2}(B B) \leq M_{2}(T)$. Since $M_{2}\left(T_{9}\right)=4 n-2<4 n-1=M_{2}(B B), M_{2}\left(T_{9}\right)<M_{2}(T)$. Now, suppose that $n_{3}(T) \leq 2$. If $n_{3}(T)=1$, then $T$ is a subdivision graph of $S_{4}$. As we illustrated in Figure 4, the subdivision graphs of $S_{4}$, are $T_{2}, T_{3}$, and $T_{4}$. It is easy to see that $M_{2}\left(T_{2}\right)=4 n-6, M_{2}\left(T_{3}\right)=4 n-5$, and $M_{2}\left(T_{4}\right)=4 n-4$. If $n_{3}(T)=2$, then $T$ is a subdivision graph of $A$. As we illustrated in Figures 4 and 5 , the subdivision graphs of $A$, are $T_{5}, T_{6}, \ldots, T_{8}, T_{10}, \ldots, T_{14}$. It is easy to see that $M_{2}\left(T_{5}\right)=4 n-4$, $M_{2}\left(T_{6}\right)=4 n-3, M_{2}\left(T_{7}\right)=M_{2}\left(T_{8}\right)=M_{2}\left(T_{9}\right)=4 n-2, M_{2}\left(T_{10}\right)=M_{2}\left(T_{12}\right)=4 n-1$, $M_{2}\left(T_{11}\right)=M_{2}\left(T_{13}\right)=4 n$, and $M_{2}\left(T_{14}\right)=4 n+1$.

Case 3: $\Delta(T)=2$. Then, $T \cong P_{n}$ and $M_{2}(T)=4 n-8$. The above deductions, lead us the following theorem.

Theorem 3.3. Suppose that $T$ is a tree with $n(\geq 11)$ vertices, except $T_{1}, T_{2}, \ldots, T_{9}$, as illustrated in Figure 4. Then, we have

$$
\begin{aligned}
M_{2}\left(T_{1}\right) & <M_{2}\left(T_{2}\right)<M_{2}\left(T_{3}\right)<M_{2}\left(T_{4}\right)=M_{2}\left(T_{5}\right)<M_{2}\left(T_{6}\right)<M_{2}\left(T_{7}\right) \\
& =M_{2}\left(T_{8}\right)=M_{2}\left(T_{9}\right)<M_{2}(T) .
\end{aligned}
$$


Acknowledgements. The authors are grateful to the referee for helpful suggestions. This work was supported by the Research Grant Khansar-CMC-002.

\section{REFERENCES}

[1] B. Borovicanin and T. A. Lampert, On the maximum and minimum Zagreb indices of trees with a given number of vertices of maximum degree, MATCH Commun. Math. Comput. Chem. 74 (2015), 81-96.

[2] J. Braun, A. Kerber, M. Meringer amd C. Rucker, Similarity of molecular descriptors: the equivalence of Zagreb indices and walk counts, MATCH Commun. Math. Comput. Chem. 54 (2005), 163-176.

[3] K. C. Das and I. Gutman, Some properties of the second Zagreb index, MATCH Commun. Math. Comput. Chem. 52 (2004), 103-112.

[4] H. Deng, A unified approach to the extremal Zagreb indices for trees, unicyclic graphs and bicyclic graphs, MATCH Commun. Math. Comput. Chem. 57 (2007), 597-616.

[5] J. Devillers and A. T. Balaban (Eds.), Topological Indices and Related Descriptors in: QSAR and QSPR, Gordon Breach, Amsterdam, 1999.

[6] M. Goubko, Minimizing degree-based topological indices for trees with given number of pendent vertices, MATCH Commun. Math. Comput. Chem. 71 (2014), 33-46.

[7] I. Gutman and N. Trinajstić, Graph theory and molecular orbitals. Total p-electron energy of alternant hydrocarbons, Chem. Phys. Lett. 17 (1972), 535-538.

[8] A. N. Habibi, T. Dhghan Nejad and A. R. Ashrafi, Extremal tetracyclic graphs whth respect to the first and second Zagreb indices, Trans. Comb. 5 (2016), 35-55.

[9] R. Lang, X. Deng and H. Lu, Bipartite graphs with the maximal value of the second Zagreb index, Bull. Malays. Math. Sci. Soc. 36(1) (2013), 1-6.

[10] S. Li and M. Zhang, Sharp upper bounds for Zagreb indices of bipartite graphs with a given diameter, Appl. Math. Lett. 24(2) (2011), 131-137.

[11] S. Li and H. Zhou, On the maximum and minimum Zagreb indices of graphs with connectivity at most k, Appl. Math. Lett. 23(2) (2010), 128-132.

[12] B. Liu, Some estimations of Zagreb indices, Util. Math. 74 (2007), 239-245.

[13] S. Nikolić, G. Kovacević, A. Milićević and N. Trinajstić, The Zagreb indices 30 years after, Croat. Chem. Acta 76 (2003), 113-124.

[14] R. Todeschini and V. Consonni, New local vertex invariants and molecular descriptors based on functions of the vertex degrees, MATCH Commun. Math. Comput. Chem. 64 (2010), 359-372.

[15] R. Todeschini, D. Ballabio and V. Consonni, Novel molecular descriptors based on functions of new vertex degrees, in: I. Gutman and B. Furtula (Eds.), Novel Molecular Structure DescriptorsTheory and Applications I, Univ. Kragujevac, Kragujevac, 2010, pp. 73-100.

[16] D. Vukicevic, S. M. Rajtmajer and N. Trinajstic, Trees with maximal second Zagreb index and prescribed number of vertices of the given degree, MATCH Commun. Math. Comput. Chem. 60 (2008), 65-70.

[17] Z. Yan and H. Liu, Sharp bounds for the second Zagreb index of unicyclic graphs, J. Math. Chem. 42 (2007), 565-574.

[18] Q. Zhao and S. Li, On the maximum Zagreb indices of graphs with $k$ cut vertices, Acta Appl. Math. 111(1) (2010), 93-106.

[19] B. Zhou and I. Gutman, Relations between Wiener, hyper-Wiener and Zagreb indices, Chem. Phys. Lett. 394 (2004), 93-95.

[20] B. Zhou, Zagreb indices, MATCH Commun. Math. Comput. Chem. 52 (2004), 113-118.

[21] B. Zhou and I. Gutman, Further properties of Zagreb indices, MATCH Commun. Math. Comput. Chem. 54 (2005), 233-239. 
1 Department of Mathematics,

Khansar Faculty of Mathematics and Computer Science,

P. O. Box 87915-85163, Khansar, IrAN

E-mail address: eliasi@math.iut.ac.ir

E-mail address: Ali797ghalavand@gmail.com 